JOURNAL OF INTEGRAL EQUATIONS

AND APPLICATIONS

Volume 7, Number 4, Fall 1995

\title{
STABILITY OF CONTINUOUS AND DISCRETE VOLTERRA INTEGRO-DIFFERENTIAL EQUATIONS BY LIAPUNOV APPROACH
}

\author{
M.R. CRISCI, V.B. KOLMANOVSKII, E. RUSSO AND A. VECCHIO
}

ABSTRACT. The Liapunov method is used to obtain stability conditions on some classes of Volterra integro-differential equations, and it is generalized for studying stability of discrete Volterra equations. As a first application of such generalization, stability conditions for the Volterra linear multistep methods, applied to a general linear system of Volterra integro-differential equations, are derived.

1. Introduction. The aim of this paper is stability investigation of Volterra integro-differential equations (VIDEs) and their discretized version.

Since universal stability conditions are not known, we should try to study stability problems for special classes of VIDEs under appropriate assumptions. These equations should also be treated as a collection of test problems which would be useful to check the stability of numerical methods.

As is known, a powerful tool to analyze stability of a problem is the direct Liapunov method. It consists of the use of suitable functionals depending on the solution of the equation under consideration. Through the construction of such functionals, in Section 2, we obtain stability conditions for different types of VIDEs. Some of these conditions, if the integral term is zero, coincide with the known stability conditions for ODEs.

In Section 3 we consider a discrete VIDE, that is, a difference equation of unbounded order. For this equation we prove a general theorem which gives stability conditions depending on the existence of suitable functions. In other words, we extend the discrete Liapunov theory to the case of unbounded order difference equations. We stress that this theorem furnishes, as in the continuous case, a powerful tool to

Received by the editors on November 9, 1994.

Copyright (c)1995 Rocky Mountain Mathematics Consortium 
investigate stability of discrete equations arising from the application of a numerical method to a continuous VIDE.

As a first realization of this approach, we consider Volterra linear multistep methods, and we obtain conditions for numerical stability formulated in terms of the coefficients of the method, of the kernel and the stepsize.

We remark that if the integral term is assumed equal to zero, the stability conditions, obtained for the one-step methods, coincide with the known ones for ordinary differential equations.

Finally, we emphasize that the aim of the paper is not only to obtain explicit stability conditions, but also to show the essence of the proposed procedures to construct Liapunov functionals and functions for Volterra continuous and discrete equations.

2. The continuous case. Consider the nonlinear system of Volterra integro-differential equations

$$
\begin{aligned}
& y^{\prime}(t)=G(t, y(t))+\int_{0}^{t} k(t, s, y(s)) d s, \quad t \geq 0, \\
& y(0)=y_{0} .
\end{aligned}
$$

Here $y(t) \in R^{n}, G:[0, \infty) \times R^{n} \rightarrow R^{n}$ and $k:[0, \infty) \times[0, \infty) \times[0, \infty) \times$ $R^{n} \rightarrow R^{n}$ are continuous functions satisfying local Lipschitz conditions with respect to $y$ and $G(t, 0) \equiv 0, k(t, s, 0) \equiv 0$ for all $t, s \geq 0$.

Though the intuitive meaning of stability is clear, much controversy exists about formal definitions. The stability of definition used below is the natural generalization of the stability definition proposed by Liapunov.

Definition 2.1. The trivial solution of equation (2.1) defined by the zero initial condition $y(0)=0$ will be called

1) stable if for any $\varepsilon>0$ there exists $\delta_{\varepsilon}$ such that $\|y(t)\| \leq \varepsilon, t \geq 0$, for any initial vector $y_{0}$ with $\left\|y_{0}\right\| \leq \delta$;

2) asymptotically stable if it is stable and $\lim y(t)=0, t \rightarrow \infty$ for all vectors $y_{0} \in D$ where $D$ is some neighborhood of the origin. Sometimes $D$ is called the domain of attraction of the trivial solution. 
In order to prove the first general theorem on stability of (2.1), assume also that the function $G(t, y)$ is continuously differentiable with respect to $y$ and

$$
\|k(t, s, y)\| \leq \kappa(t, s)\|y\|
$$

where $\kappa(t, s)$ is a continuous nonnegative function. Moreover, let us denote by $g(t, y)$ the Jacobian matrix corresponding to $G(t, y)$ (i.e., $g(t, x)=\partial G(t, y) / \partial y)$ and by $\gamma(g(t, y))$ the logarithmic norm of the matrix $g(t, y)$.

The proof of the next theorem is based on the following standard lemma contained in any stability course on functional-differential equations (see, e.g., [3, p. 103]).

Before reporting this lemma, the definitions of positive definite and decrescent functional must be recalled. These definitions can be found in $\left[3\right.$, p. 102]. Denote by $y_{t}$ the whole trajectory of solution of the problem $(2.1),(2.2)$ on the interval $[0, t]$, i.e., $y_{t}=y(t+\theta)$, where, for any $t$, the argument $\theta$ takes all the values $-t \leq \theta \leq 0$.

Let $\omega_{i}(r)$ be scalar continuous increasing functions such that $\omega_{i}(0)=$ 0 .

Definition 2.2. A functional $V\left(t, y_{t}\right)$ is called positive definite (decrescent) if there exists a function $\omega_{1}(r)$ (function $\omega_{2}(r)$ ) such that $V\left(t, y_{t}\right) \geq \omega_{1}(\|y(t)\|)\left(\operatorname{such}\right.$ that $V\left(t, y_{t}\right) \leq \omega_{2}\left(\sup _{-t \leq \theta \leq 0}\|y(t+\theta)\|\right)$.

Lemma 2.1. The trivial solution of (2.1) is asymptotically stable if there exists a scalar continuous functional $V\left(t, y_{t}\right)$ which, for any solution $y(t)$ of the problem (2.1), (2.2), belonging to $D$ for all $t$, is positive definite, decrescent and its right upper total derivative denoted as $d^{+} V\left(t, y_{t}\right) / d t$ is negative definite.

Theorem 2.2. Assume that

$$
a>\sup _{t>0} \int_{0}^{\infty} \kappa(t+s, t) d s
$$

where

$$
a=-\sup _{t \geq 0, y \in D} \gamma(g(t, y))
$$


396 M.R. CRISCI, V.B. KOLMANOVSKII, E. RUSSO AND A. VECCHIO

and $\kappa$ is defined in (2.3). Then the trivial solution of (2.1) is asymptotically stable.

Proof. Consider the functional $V\left(t, y_{t}\right)$

$$
V=\|y(t)\|+\int_{0}^{\infty} d s \int_{t-s}^{t} \kappa(\tau+s, \tau)\|y(\tau)\| d \tau
$$

where $y(\tau) \equiv 0$ for $\tau<0$.

It is clear that the functional (2.6) is positive definite and decrescent. To verify that $d^{+} V / d t$ is negative definite, we obtain

$$
\begin{aligned}
\frac{d^{+} V}{d t}= & \frac{d^{+}\|y(t)\|}{d t}-\int_{0}^{t} \kappa(t, t-s)\|y(t-s)\| d s \\
& +\|y(t)\| \int_{0}^{\infty} \kappa(t+s, t) d s
\end{aligned}
$$

For any norm $\|\cdot\|$ in $R^{n}$ (see, e.g., [2, p. 461])

$$
\frac{d^{+}\|y(t)\|}{d t}=Q\left[y(t), y^{\prime}(t)\right]
$$

where the function $Q(u, v)$ of two independent arguments, $u, v \in R^{n}$, is defined by the relation

$$
Q(u, v)=\lim _{h \rightarrow 0^{+}} \frac{1}{h}[\|u+h v\|-\|u\|] .
$$

From (2.8) and (2.9), it follows that

$$
\frac{d^{+}\|y(t)\|}{d t}=\lim _{h \rightarrow 0^{+}} \frac{1}{h}\left[\left\|y(t)+h y^{\prime}(t)\right\|-\|y(t)\|\right] .
$$

Substituting in (2.10) the right side of (2.1), instead of $y^{\prime}(t)$, we get

$$
\begin{aligned}
\frac{d^{+}|| y(t) \|}{d t} \leq & \lim _{h \rightarrow 0^{+}} \frac{1}{h}[\| y(t)+h G(t, y(t)\|-\| y(t) \|] \\
& +\left\|\int_{0}^{t} k(t, s, y(s)) d s\right\| \\
\leq & Q\left[y(t), G(t, y(t)]+\int_{0}^{t} \kappa(t, s)\|y(s)\| d s .\right.
\end{aligned}
$$


But

$$
G(t, y(t))=\left[\int_{0}^{1} g(t, s y(t)) d s\right] y(t)
$$

hence

$$
\begin{aligned}
\frac{d^{+}|| y(t) \|}{d t} \leq & Q\left[y(t), \int_{0}^{1} g(t, s y(t)) d s y(t)\right] \\
& +\int_{0}^{t} \kappa(t, s)\|y(s)\| d s
\end{aligned}
$$

Now taking into account $[\mathbf{2}$, p. 462$]$ that for any matrix $g$ the logarithmic norm satisfies $\gamma(g(t, y))=\sup _{x \in R^{n},\|x\| \neq 0}\|x\|^{-1} Q[x, g(t, y) x]$, we obtain

$$
Q\left[y(t), \int_{0}^{1} g(t, s y(t)) d s y(t)\right] \leq \gamma\left[\int_{0}^{1} g(t, s y(t)) d s\right]\|y(t)\| .
$$

Further, from (2.5) and convexity of the logarithmic norm, it follows that

$$
\gamma\left[\int_{0}^{1} g(t, s y(t)) d s\right] \leq \int_{0}^{1} \gamma(g(t, s y(t))) d s \leq-a .
$$

Consequently, because of (2.12),

$$
Q\left[y(t), \int_{0}^{1} g(t, s y(t)) d s y(t)\right] \leq-a\|y(t)\| .
$$

This inequality and (2.11) mean that

$$
\frac{d^{+}\|y(t)\|}{d t} \leq-a\|y(t)\|+\int_{0}^{t} \kappa(t, t-s)\|y(t-s)\| d s ;
$$

comparing (2.7) and (2.13) we see that

$$
\frac{d^{+} V}{d t} \leq-\left(a-\sup _{t>0} \int_{0}^{\infty} \kappa(t+s, t) d s\right)\|y(t)\|<0 .
$$

Relation (2.14) shows also that $V\left(t, y_{t}\right) \leq V\left(0, y_{0}\right)$. So the functional (2.6) satisfies all the conditions of Lemma 2.1, and the theorem is proved. 
We observe that if the kernel $k$ is assumed equal to zero then (2.4) and (2.5) coincide with the known stability condition for ODEs $[4, \mathrm{p}$. 269].

Theorem 2.1 is quite general, and it is interesting not only for the stability condition there derived, but also because it illustrates the Liapunov approach to general integro-differential equations.

Using the same arguments as in the proof of Theorem 2.1, we can establish the two following results.

Consider the VIDE

$$
y^{\prime}(t)=G_{1}(t, y(t)) y(t)+G_{2}(t, y(t)) \int_{0}^{t} k(t, s, y(s)) d s, \quad t \geq 0
$$

Suppose $G_{1}:[0, \infty) \times R^{n}, G_{2}:[0, \infty) \times R^{n} \rightarrow R^{n \times n}$ are bounded and continuous, $G_{i}(t, 0) \equiv 0$ and the kernel $k$ is such that

$$
\left\|G_{2}(t, y(t)) k(t, s, y(s))\right\| \leq \kappa_{1}(t, s)\|y(s)\|
$$

with $\kappa_{1}(t, s)$ a continuous nonnegative function.

Theorem 2.2. Assume that

$$
a_{1}>\sup _{t>0} \int_{0}^{\infty} \kappa_{1}(t+s, t) d s
$$

where

$$
a_{1}=-\sup _{t \geq 0, y \in D} \gamma\left(G_{1}(t, y)\right)
$$

Then the trivial solution of equation (2.15) is asymptotically stable.

Proof. The theorem can be proved analogously to Theorem 2.1 by taking the same functional (2.6) and changing $\kappa$ into $\kappa_{1}$.

An interesting application of this corollary is given in the following example. 
Example 2.1. Let us consider the two-dimensional VIDE of the form (2.15) with

$$
\begin{gathered}
G_{1}\left(\begin{array}{cc}
-a_{1} & \alpha(t, y(t)) \\
-\alpha(t, y(t)) & -a_{1}
\end{array}\right), \quad G_{2}=I, \\
k=\left(\begin{array}{cc}
e^{-q(t-s)} & 0 \\
0 & e^{-q(t-s)}
\end{array}\right) y(t)
\end{gathered}
$$

where $a_{1}$ and $q$ are positive constants, the function $\alpha:[0, \infty) \times R^{2} \rightarrow R$ is continuous and bounded, $y=\left(y_{1}, y_{2}\right)$ and $I$ is the identity matrix.

Take the Euclidean norm in $R^{2}$. Then the logarithmic norm of the matrix $G_{1}$ is equal to the maximal eigenvalue of the symmetric matrix $\left(G_{1}+G_{1}^{T}\right) / 2$, i.e., $\gamma\left(G_{1}\right)=-a_{1}$. Further, since in this case

$$
\|k\| \leq \exp (-q(t-s))\|y(s)\|,
$$

the asymptotic stability condition of Corollary 2.1 applied to equation $(2.17)$ is

$$
a_{1}>\sup _{t>0} \int_{0}^{t} e^{-q s} d s=\frac{1}{q} \sup _{t>0}\left[1-e^{-q t}\right]=\frac{1}{q} .
$$

We observe that if $q$ tends to infinity then the asymptotic stability condition (2.18) turns into $a_{1}>0$, which can be obtained also by the following arguments. If $q \rightarrow \infty$, then the VIDE under consideration becomes the ordinary differential equation

$$
y^{\prime}(t)=G_{1}(t, y(t)) y(t)
$$

which can be easily proved to be exponentially stable under the same hypothesis $a_{1}>0$. For the proof it is sufficient to use the Liapunov direct method applied to the function $V(t, y(t))=\|y(t)\|$.

Moreover, we want to stress that the stability condition (2.18) does not depend on the function $\alpha(t, y)$.

From Theorem 2.1 the following corollary, which will be useful in Section 3, can also be immediately derived.

Corollary 2.1. The trivial solution of the VIDE system

$$
y^{\prime}(t)=A(t) y(t)+\int_{0}^{t} k(t, s) y(s) d s
$$


with

$$
\sup _{t>0} \gamma(A(t))<-\int_{0}^{\infty}\|k(t+s, s)\| d s
$$

is asymptotically stable.

In the remaining part of this section we want to illustrate how, depending on the type of VIDE we are considering, more appropriate functional $V$ can be found, leading to different sufficient stability conditions. For example, in the sequel, three different functionals $V$ (related to three different classes) of VIDEs, are derived. We want to add that another functionals for investigating stability of Volterra equations was proposed in [5].

2.1. Second order equations. Let us consider the two-dimensional VIDE system

$$
\left\{\begin{array}{l}
x^{\prime}(t)=y(t) \\
y^{\prime}(t)=-\phi(t, y(t))-f(x(t))+\int_{0}^{t} k(t, t-s) y(t-s) d s \quad t \geq 0
\end{array}\right.
$$

where $\phi:[0, \infty) \times R \rightarrow R, k:[0, \infty) \times[0, \infty) \rightarrow R$, and $f: R \rightarrow R$ are continuous functions $\phi(t, 0) \equiv 0, f(0)=0$. Then the following theorem holds.

Theorem 2.3. Assume that

$$
\begin{gathered}
2 z \phi(t, z) \geq z^{2}\left[\int_{0}^{t}|k(t, t-s)| d s+\int_{0}^{\infty}|k(t+s, t)| d s\right] \\
t \geq 0, \quad z \in(-\infty, \infty) \\
z f(z)>0, \quad \text { if }|z|>0
\end{gathered}
$$

then the trivial solution of equation (2.21) is stable.

Proof. For the stability investigation of the trivial solution of (2.21) 
we take the functional

$$
\begin{aligned}
V= & 2 \int_{0}^{x(t)} f(s) d s+y^{2}(t) \\
& +\int_{0}^{\infty} d s \int_{t-s}^{t}|k(\tau+s, \tau)| y^{2}(\tau) d \tau
\end{aligned}
$$

where $y(\tau)=0, \tau<0$.

Calculating $V^{\prime}$, we obtain

$$
V^{\prime} \leq-2 y \phi(t, y)+y^{2}\left[\int_{0}^{t}|k(t, t-s)| d s+\int_{0}^{\infty} \mid k(t+s, t) d s\right]
$$

and the thesis follows. $\quad \square$

Remark 2.1. We observe that in this case the stability conditions are obtained without any assumptions about differentiability of the functions $\phi(t, y)$ and $f(x)$ which were essential for Theorem 2.1.

\subsection{Scalar equations.}

A) Linear equations with differentiable kernel. Let us consider the scalar VIDE

$$
x^{\prime}(t)=-a x(t)-\int_{0}^{t} k(t-s) x(s) d s, \quad t>0
$$

where $a>0$ is a constant and the function $k:[0, \infty) \rightarrow R$ is continuously differentiable. For such an equation, the following result can be proved:

Theorem 2.4. If

$$
a k(0)>(1+a) \int_{0}^{\infty}|\alpha(s)| d s, \quad a>\int_{0}^{\infty}|\alpha(s)| d s,
$$

then the trivial solution of equation (2.22) is asymptotically stable. 
Proof. Differentiating both sides of $(2.22)$ in $t$, and putting $y=x^{\prime}$, we get the system

$$
\left\{\begin{array}{l}
x^{\prime}(t)=y(t) \\
y^{\prime}(t)=-a y(t)-b x(t)-\int_{0}^{t} \alpha(t-s) x(s) d s
\end{array}\right.
$$

with $b=k(0)$ and $\alpha(s)=k^{\prime}(s)$. Continue $x(t)$ for $(t<0)$ by setting $x(t) \equiv 0, t<0$, and introduce the functionals

$$
\begin{aligned}
V & =V_{1}+V_{2} \\
V_{1} & =2 b x^{2}(t)+y^{2}(t)+(y(t)+a x(t))^{2}, \\
V_{2} & =(2+a) \int_{0}^{\infty}|\alpha(s)| d s \int_{t-s}^{t} x^{2}(\tau) d \tau .
\end{aligned}
$$

By applying Cauchy's inequality, we get

$$
V^{\prime} \leq 2 x^{2}(t)\left[-a b+(1+a) \int_{0}^{\infty}|\alpha(s)| d s\right]+2 y^{2}(t)\left[-a \int_{0}^{\infty}|\alpha(s)| d s\right]
$$

and the hypotheses $(2.23)$ assure $V^{\prime}<0$ which completes the proof. 口

Remark 2.2. We note that if the kernel $k$ is a constant, then stability conditions (2.23) turn into $a>0, k>0$, and they are necessary and sufficient conditions which coincide with the known stability conditions for the basic test equation [1, p. 418]

$$
y^{\prime}(t)=\lambda y(t)+\mu \int_{0}^{t} y(s) d s .
$$

We also stress that such result concerning the basic test equation cannot be obtained directly by Theorem 2.1 because the kernel in (2.24) is not $L^{1}$.

B) Linear equation with general kernel. Let us consider the scalar VIDE

$$
y^{\prime}(t)=-\int_{0}^{\infty} k(t, s) y(t-s) d s, \quad t \geq 0
$$


with the initial condition

$$
y(\theta)=\phi(\theta), \quad \theta \leq 0
$$

where $\phi:(-\infty, 0] \rightarrow R$ is a given, continuous bounded function, and $k:[0, \infty) \times[0, \infty) \rightarrow R$ is a continuous function. Then the following result can be established:

Theorem 2.5. Let us assume that

$$
\begin{gathered}
A(t)=\int_{0}^{\infty} k(t+s, s) d s \geq 0, \quad \sup _{t \geq 0} \int_{0}^{\infty}|k(t, s)| d s<\infty \\
\alpha>0 \\
\alpha_{1}=\sup _{t \geq 0} \int_{0}^{\infty} d s \int_{t-s}^{t}|k(\tau+s, s)| d \tau<1
\end{gathered}
$$

then the trivial solution of the VIDE (2.25) is uniformly asymptotically stable.

Proof. To obtain stability conditions of the trivial solution of equation (2.25) with respect to a disturbance of the initial condition, let us introduce the functional

$$
\begin{aligned}
V & =V_{1}+V_{2} \\
V_{1} & =\left[y(t)-\int_{0}^{\infty} d s \int_{t-s}^{t} k(\tau+s, s) x(\tau) d \tau\right]^{2}, \\
V_{2} & =\int_{0}^{\infty} d s \int_{t-s}^{t} A\left(s_{1}+s\right) d s_{1} \int_{s_{1}}^{t}|k(\tau+s, s)| y^{2}(\tau) d \tau
\end{aligned}
$$

where all integrals are assumed to be absolutely convergent. After computing $V_{1}^{\prime}$ and $V_{2}^{\prime}$, we get

$$
\begin{gathered}
V^{\prime} \leq-\alpha x^{2}(t) \\
\alpha=\inf _{t \geq 0}\left[2 A(t)-A(t) \int_{0}^{\infty} d s \int_{t-s}^{t}|k(\tau+s, s)| d \tau\right. \\
\left.-\int_{0}^{\infty}|k(t+s, s)| d s \int_{t-s}^{t} A\left(s_{1}+s\right) d s_{1}\right] .
\end{gathered}
$$


404 M.R. CRISCI, V.B. KOLMANOVSKII, E. RUSSO AND A. VECCHIO

From (2.29) and (2.27), it follows that

$$
\int_{0}^{\infty} y^{2}(s) d s<\infty
$$

and also

$$
\left|y(t)-\int_{0}^{\infty} d s \int_{t-s}^{t} k(\tau+s, s) y(\tau) d \tau\right| \leq C<\infty, \quad \forall t \geq 0 .
$$

Inequalities (2.28) and (2.31) mean that

$$
\sup _{t \geq 0}|y(t)|<\infty .
$$

From here and (2.26), one can conclude that

$$
\sup _{t \geq 0}\left|y^{\prime}(t)\right|<\infty
$$

Inequalities (2.30), (2.32) lead to

$$
\lim _{t \rightarrow \infty} y(t)=0
$$

which completes the proof.

Remark 2.3. Comparing the stability conditions (2.26), (2.27) and (2.28) with the ones obtained in Theorem 2.1, we note that no assumption about "instantaneous feedback" is requested. The presence of such a term (function $G(t, y(t))$ ) was on the contrary essential for application of general stability criteria from Theorem 2.1.

3. The discrete case. In this section we treat the stability of the discrete Volterra equations.

We start by generalizing the Liapunov theorem for difference equations of unbounded order

$$
\begin{gathered}
y_{i+1}=F\left(i, y_{0}, y_{1}, \ldots, y_{i}\right), \quad i \geq 0, \quad y_{i} \in R^{n}, \\
F(i, 0,0, \ldots, 0) \equiv 0
\end{gathered}
$$


and for the sake of completeness, firstly we report the following definitions corresponding to Definition 2.1 in the continuous case.

Definition 3.1. The solution $y_{i}$ of (3.1) is said to be

1) stable if for all $\varepsilon>0$ there exists a $\delta=\delta_{\varepsilon}>0$ such that $\left\|y_{i}\right\|<\varepsilon$, $i>0$ for any initial vector $y_{0}$ with $\left\|y_{0}\right\|<\delta$;

2) asymptotically stable if it is stable and $\lim _{i \rightarrow \infty}\left\|y_{i}\right\|=0$ for all vectors $y_{0} \in D$ where $D$ is some neighborhood of the origin.

Now the following general theorem which can be considered as a generalization of the corresponding theorem for equations with finite delay [3], can be proved. Let $\omega_{i}(r)$ be scalar continuous increasing functions such that $\omega_{i}(0)=0$.

Theorem 3.1. If there exists a scalar function $V_{i}\left(y_{0}, \ldots, y_{i}\right)$ continuous with respect to all the variables $y_{0}, \ldots, y_{i}, \ldots$, such that

i) $V_{0}(0)=0$

ii) $V_{i}\left(y_{0}, \ldots, y_{i}\right) \geq \omega_{1}\left(\left\|y_{i}\right\|\right)$

iii) $\Delta V_{i}=V_{i+1}\left(y_{0}, \ldots, y_{i}, y_{i+1}\right)-V_{i}\left(y_{0}, \ldots, y_{i}\right) \leq 0$

then the solution of (3.1) is stable. If, in addition,

iv) $\Delta V_{i} \leq-\omega_{2}\left(\left\|y_{i}\right\|\right)$

then the solution of (3.1) is asymptotically stable.

Proof. Let us consider an arbitrary positive number $\varepsilon>0$. For the continuity of the function $V_{0}\left(y_{0}\right)$ there exists a positive number $\delta=\delta_{\varepsilon}$ such that $V_{0}\left(y_{0}\right)<\omega_{1}(\varepsilon)$ if $\left\|y_{0}\right\|<\delta$. Then by ii) and iii) there results

$$
\omega_{1}\left(\left\|y_{i}\right\|\right)<V_{i}\left(y_{0}, \ldots, y_{i}\right) \leq V_{0}\left(y_{0}\right)<\omega_{1}(\varepsilon) .
$$

So, since $\omega_{1}(0)=0$ and $\omega_{1}$ is an increasing continuous function, (3.2) implies $\left\|y_{i}\right\|<\varepsilon$. Therefore we have proved that

$$
\forall \varepsilon>0 \quad \exists \delta=\delta_{\varepsilon} \text { s.t. if }\left\|y_{0}\right\|<\delta, \quad\left\|y_{i}\right\|<\varepsilon, \quad \forall i \geq 0
$$

which is equivalent to the stability of the solution. 
Let us prove the asymptotical stability under the additional assumption iv). Show that for arbitrary initial condition $y_{0}$ the corresponding solution $y_{i}$ of (3.1) tends to zero as $i \rightarrow \infty$. Assume the opposite, i.e., there exists a positive number $\varepsilon>0$ and an infinite sequence $\left\{k_{i}\right\}$, such that

$$
\left\|y_{k_{i}}\right\|>\varepsilon, \quad i \geq 0 .
$$

Let us represent $V_{i}\left(y_{0}, \ldots, y_{i}\right)$ in the form

$$
V_{i}\left(y_{0}, \ldots, y_{i}\right)=V_{0}\left(y_{0}\right)+\sum_{l=0}^{i-1} \Delta V_{l \notin\left\{k_{i}\right\}}+\sum_{l=0}^{i-1} \Delta V_{l \in\left\{k_{i}\right\}},
$$

but by virtue of iv)

$$
\Delta V_{l} \leq-\omega_{2}\left(\left\|y_{l}\right\|\right)<-\omega_{2}(\varepsilon), \quad \forall l \in\left\{k_{i}\right\} .
$$

Hence, recalling also that $\Delta V_{l} \leq 0$ for all $l \geq 0$, we get

$$
V_{i}\left(y_{0}, \ldots, y_{i}\right) \leq V_{0}\left(y_{0}\right)-M(i) \omega_{2}(\varepsilon)
$$

where $M(i)$ is the number of the terms of the set $\left\{k_{i}\right\}$ such that $k_{i} \leq i-1$. Finally, take into consideration that because of our assumption $M(i) \rightarrow \infty$ as $i \rightarrow \infty$. It means that $V_{i}\left(y_{0}, \ldots, y_{i}\right) \rightarrow-\infty$ as $i \rightarrow \infty$ which contradicts ii). Consequently, for any solution $y_{i}$ of (3.1) we get $y_{i} \rightarrow 0, i \rightarrow \infty$.

Now since the aim of this section is to give a first example of how the Liapunov approach can work on the discrete VIDE, we use the previous theorem for studying the numerical stability of some Volterra linear multistep (VLM) methods.

Let us consider the $n$-dimensional system of VIDE (2.19) with the assumption (2.20) and apply a $k$-step VLM method [6, p. 182]. The numerical solution $y_{i} \approx y\left(t_{i}\right)$ where $t_{i}=i$ satisfies the following difference equation system

$$
\sum_{j=0}^{k} \alpha_{j} y_{i+j}=h \sum_{j=0}^{k} \beta_{j} A\left(t_{i+j}\right) y_{i+j}+h \sum_{j=0}^{k} \beta_{j} F\left(t_{i+j}\right), \quad i \geq k
$$


where $\alpha_{j}$ and $\beta_{j}, j=0, \ldots, k$, are the coefficients of the VLM method under consideration, and the lag term $F(t)$ is an approximation of $\int_{0}^{t} k(t, s, y(s)) d s$, i.e.,

$$
F\left(t_{i}\right)=h \sum_{r=0}^{i} w_{i, r} k\left(t_{i}, t_{r}\right) y_{r}
$$

Here $w_{i, r}$ are the coefficients of suitable quadrature formulas.

Before proving the next theorem we add to (3.3) the natural assumption

$$
y_{r}=0, \quad r<0
$$

and we need the following notations

$$
R_{i}=\left[\alpha_{k} I-h \beta_{k} A\left(t_{i+k}\right)-h^{2} \beta_{k} w^{*} k\left(t_{i+k}, t_{i+k}\right)\right]^{-1} \in R^{n \times n}
$$

where $w^{*}=w_{i+k, i+k}$ is the last coefficient of the quadrature formula, which is usually independent of $i$.

$$
T_{j, r}=-\alpha_{j} I+h \beta_{j} A\left(t_{r}\right) \in R^{n \times n}
$$

where $I$ is the $n \times n$ identity matrix.

\section{Theorem 3.2. If}

(a) $\left|w_{i, r}\right| \leq w, i, r \geq 0$,

(b) $\quad-1+\sum_{j=0}^{k-1}|| R_{i+k-1-j}\left\||| T_{j, i+k-1}\right\|+h^{2} w \sum_{j=0}^{k}\left|\beta_{j}\right| \sum_{\nu=0}^{\infty}$ $\left\|R_{i+k-1-j+\nu}|||| k\left(t_{i+k-1+\nu}, t_{i+k-1}\right)\right\| \leq 0, i \geq 0$,

then the solution $y_{i}, i \geq 0$, of (3.3) is stable.

Proof. By computing $y_{i+k}$ from (3.3) we obtain

$$
\begin{aligned}
y_{i+k}= & R_{i} T_{k-1, i+k-1} y_{i+k-1}+R_{i} \sum_{j=0}^{k-2} T_{j, i+j} y_{i+j} \\
& +h^{2} R_{i} \sum_{j=0}^{k} \beta_{j} \sum_{r=0}^{q} w_{i+j, r} k\left(t_{i+j}, t_{r}\right) y_{r}
\end{aligned}
$$


408 M.R. CRISCI, V.B. KOLMANOVSKII, E. RUSSO AND A. VECCHIO

where

$$
q=\min \{i+j, i+k-1\}
$$

Then consider a norm $\|\cdot\|$ in $R^{n}$ and the following functions:

$$
\begin{gathered}
V_{i}\left(y_{0}, \ldots, y_{i}\right)=G_{i}\left(y_{0}, \ldots, y_{i}\right)+H_{i}\left(y_{0}, \ldots, y_{i}\right)+L_{i}\left(y_{0}, \ldots, y_{i}\right) \\
G_{i+k-1}\left(y_{0}, \ldots, y_{i+k-1}\right)=\left\|y_{i+k-1}\right\|, \\
H_{i+k-1}\left(y_{0}, \ldots, y_{i+k-1}\right)=\sum_{j=0}^{k-2} \sum_{r=i+j}^{i+k-2}\left\|R_{r-j}\right\|\left\|T_{j, r}\right\|\left\|y_{r}\right\|, \\
L_{i+k-1}\left(y_{0}, \ldots, y_{i+k-1}\right) \\
=h^{2} \sum_{j=0}^{k}\left|\beta_{j}\right| \sum_{\nu=q_{1}}^{\infty} \sum_{r=i+j-\nu}^{i+k-2}\left\|R_{r-j+\nu}\right\|\left\|k\left(t_{r+\nu}, t_{r}\right)\right\|\left\|y_{r}\right\|,
\end{gathered}
$$

with

$$
q_{1}=\max \{0,2-k+j\} .
$$

It is clear that $V$ verifies both i) and ii) of Theorem 3.1. Now in order to compute $\Delta V$, we compute separately $\Delta G, \Delta H$ and $\Delta L$.

$$
\Delta G_{i+k-1}=|| y_{i+k}\|-\| y_{i+k-1} \|
$$

and from (3.6)

$$
\begin{aligned}
\Delta G_{i+k-1} \leq & {\left[\left\|R_{i} T_{k-1, i+k-1}\right\|-1\right]\left\|y_{i+k-1}\right\| } \\
& +\sum_{j=0}^{k-2}\left\|R_{i}\right\|\left\|T_{j, i+j}\right\|\left\|y_{i+j}\right\| \\
& +h^{2} w \sum_{j=0}^{k}\left|\beta_{j}\right| \sum_{r=0}^{q}\left\|R_{i}\right\|\left\|k\left(t_{i+j}, t_{r}\right)\right\|\left\|y_{r}\right\|, \\
\Delta H_{i+k-1}= & \sum_{j=0}^{k-2}\left[\left\|R_{i+k-1-j}\right\|\left|\left\|T_{j, i+k-1}\right\|\right| \mid y_{i+k-1} \|\right. \\
& \left.-\left\|R_{i}\right\|\left\|T_{j, i+j}\right\| y_{i+j} \|\right],
\end{aligned}
$$




$$
\begin{aligned}
\Delta L_{i+k-1} & =h^{2} w \sum_{j=0}^{k}\left|\beta_{j}\right| \sum_{\nu=q_{1}}^{\infty}\left[|| R_{i+k-1-j+\nu} \|\right. \\
& || k\left(t_{i+k-1+\nu}, t_{i+k-1}\right)\|\mid\| y_{i+k-1} \| \\
& \left.-\left\|R_{i}\right\|\left\|k\left(t_{i+j}, t_{i+j-\nu}\right)\right\|\left\|y_{i+j-\nu}\right\|\right]
\end{aligned}
$$

Here $q_{2}=\max \{0,1-k+j\}$ can be substituted for $q_{1}$, and putting $i+j-\nu=r(3.8)$ becomes

$$
\begin{aligned}
& \Delta L_{i+k-1}= h^{2} w\left[\sum_{j=0}^{k}\left|\beta_{j}\right| \sum_{\nu=q_{1}}^{\infty}\left\|R_{i+k-1-j+\nu}\right\|\right. \\
&\left\|k\left(t_{i+k-1+\nu}, t_{i+k-1}\right)\right\||| y_{i+k-1} \| \\
&\left.-\sum_{j=0}^{k}\left|\beta_{j}\right| \sum_{r=i+j-q_{2}}^{-\infty}\left\|R_{i}\right\|\left\|k\left(t_{i+j}, t_{r}\right)\right\|\left\|y_{r}\right\|\right] .
\end{aligned}
$$

Taking into account that $i+j-q_{2}=q$ and $\left(3.3^{\prime}\right)$, there results

$$
\begin{aligned}
\Delta V \leq & {\left[-1+\sum_{j=0}^{k-1}\left\|R_{i+k-1-j}\right\|\left\|T_{j, i+k-1}\right\|\right.} \\
& +h^{2} w \sum_{j=0}^{k}\left|\beta_{j}\right| \sum_{\nu=q_{1}}^{\infty}\left\|R_{i+k-1-j+\nu}\right\| \\
& \left.\left\|k\left(t_{i+k-1+\nu}, t_{i+k-1}\right)\right\|\right]\left\|y_{i+k-1}\right\|,
\end{aligned}
$$

and by hypothesis b) we get $\Delta V \leq 0$. Finally, by choosing $\omega(x)=|x|$, we have that the difference equation (3.3) satisfies all the hypotheses of Theorem 3.1 and therefore its solution $\left\{y_{i}\right\}$ is stable.

As can be easily seen, the auxiliary function $V_{i}\left(y_{0}, \ldots, y_{i}\right)$ introduced in (3.7) is the discrete analogue of the functional $V(t)$ in Theorem 2.1.

Of course, also in the discrete case different functions $V$ lead to different sufficient stability conditions.

Moreover, we note that stability condition b) derived in Theorem 3.2 becomes more tractable for particular classes of VIDEs. 
Remark 3.1. Let us assume that $A(t) \equiv A$ and $k(t, s)=k(t-s)$ in the problem (2.19). Then condition b) is independent of $i$ and we get

$$
-1+\|R\|\left|\sum_{j=0}^{k-1}\left\|T_{j}\right\|+h^{2} w\right|\left|R\left\|\left|\sum_{j=0}^{k}\right| \beta_{j} \mid \sum_{\nu=q_{1}}^{\infty}\right\| k\left(t_{\nu}\right) \|\right] \leq 0
$$

where

$$
\begin{gathered}
R=\left[\alpha_{k} I-h \beta_{k} A-h^{2} \beta_{k} w^{*} k(0)\right]^{-1}, \\
T_{j}=-\alpha_{j} I+h \beta_{j} A .
\end{gathered}
$$

Remark 3.2. In the particular case of the scalar VIDE

$$
y^{\prime}(t)=\lambda y(t)+\int_{0}^{t} k(t-s) y(s) d s, \quad \lambda<0,
$$

the stability condition (3.9) reads

$$
-1+\frac{\sum_{j=0}^{k-1}\left|-\alpha_{j}+h \lambda \beta_{j}\right|+h^{2} w \sum_{j=0}^{k}\left|\beta_{j}\right| \sum_{\nu=0}^{\infty}\left|k\left(t_{\nu}\right)\right|}{\left|\alpha_{k}-h \lambda \beta_{k}-h^{2} \beta_{k} w^{*} k(0)\right|} \leq 0
$$

We add that if the kernel $|k(s)|$ is not increasing, the infinite summation appearing in (3.10) can be surely bounded. Namely,

$$
h \sum_{\nu=0}^{\infty}\left|k\left(t_{\nu}\right)\right| \leq \int_{0}^{\infty}|k(s)| d s+h|k(0)| .
$$

Remark 3.3. We want to compare (3.10) with the known stability condition for the ODE linear multistep methods characterized by the same coefficients $\alpha_{j}, \beta_{j}$. For this purpose, let us apply Theorem 3.2 to the classical test equation

$$
y^{\prime}=\lambda y, \quad \operatorname{Re}(\lambda) \leq 0 .
$$

In such a case b) becomes

$$
\left(\sum_{j=0}^{k-1}\left|\alpha_{j}-h \lambda \beta_{j}\right|\right) /\left|\alpha_{k}-h \lambda \beta_{k}\right| \leq 1,
$$


and we observe that if (3.11) is true for all $h \lambda \leq 0$, then the corresponding ODE linear multistep method is $A$-stable. Moreover, for the one-step methods, (3.11) coincides with the known stability conditions [4, p. 70].

Finally we observe that the stability condition derived in Theorem 3.2 has an interest more theoretical than practical, since it represents, to the best of our knowledge, the first result obtained from the application of Liapunov theory to the study of stability on numerical methods for VIDEs. Since such a theory is a powerful method for studying stability of continuous VIDEs, we hope that this tool can in the future be used to also give more significant results in the numerical case.

\section{REFERENCES}

1. H. Brunner and P.J. van der Houwen, The numerical solution of Volterra equations, CWI Monograph, Amsterdam, 1986.

2. B.F. Bylov, K.E. Vinograd, D.M. Grobman and V.V. Nemytzki, Theory of Liapunov exponents, Nauka, Moscow, 1966.

3. V.B. Kolmanovskii and A.D. Myshkis, Applied theory of functional differential equations, Kluwer Academic Publishers, Dordrecht, 1992.

4. J.D. Lambert, Numerical methods for ordinary differential systems, John Wiley \& Sons, New York, 1991.

5. J.J. Levin and J.A. Nohel, Note on a non-linear Volterra equation, Proc. Amer. Math. Soc. 14 (1963), 924-929.

6. P. Linz, Analytical and numerical methods for Volterra equations, SIAM, Philadelphia, 1985.

Dip. di Mat. e Appl., Complesso Monte S. Angelo, Via Cintia, 80126 NAPOLI, ITALY

Moscow Inst. of Electronic and Math., Moscow, Russia, Novospasskit, $3-1-20$

Dip. di Mat. e Appl., Complesso Monte S. Angelo, Via Cintia, 80126 NAPOLI, ITALY

Ist. per Applicazioni della Matematica, CNR, Via P. Castellino, 111, NAPOLI, ITALY 